\title{
In-situ Electron Diffraction Studies of Sodium Electrochemistry in $\mathbf{M o S}_{2}$
}

Jinsong $\mathrm{Wu}^{1,2}$, Qianqian $\mathrm{Li}^{1,2}$, Zhenpeng $\mathrm{Yao}^{2}$, Sagar Mitra ${ }^{3}$, Shiqiang $\mathrm{Hao}^{2}$, Tuhin Subhra Sahu ${ }^{3}$, Yuan $\mathrm{Li}^{1,2}$, Christopher M. Wolverton ${ }^{2}$ and Vinayak P. Dravid ${ }^{1,2}$

1. NUANCE Center, Northwestern University, Evanston, IL, United States

2. Department of Materials Science and Engineering, Northwestern University, Evanston, IL, United States

${ }^{3 .}$ Department of Energy Science and Engineering, Indian Institute of Technology Bombay, Powai, Mumbai, India.

$\mathrm{MoS}_{2}$ is a promising electrode materials for sodium-ion batteries. In the structure of $\mathrm{MoS}_{2}$, there is adequate "space" between the $\mathrm{MoS}_{2}$ layers interconnected with weak van der Waals force to accommodate $\mathrm{Na}$ ions during charging. It turns out that $\mathrm{MoS}_{2}$ allows $\mathrm{Na}$ ions to intercalate therein without a significant volume expansion (1); which enables $\mathrm{MoS}_{2}$ to be a promising electrode material for rechargeable batteries (2). However, the number of the electrons can be accommodated in the S-Mo$\mathrm{S}$ layer is limited while the structural framework remains stable. It has been shown that up to 1.5 electrons can be stored per unit formula in $\mathrm{MoS}_{2}$ before the layered structure collapses (3). Meanwhile, there is a structural transition between trigonal $2 \mathrm{H}$ - and octahedral $1 \mathrm{~T}-\mathrm{AMoS}_{2}(\mathrm{~A}=\mathrm{Li}, \mathrm{Na}, \mathrm{K}$, etc. $)$ accompanied by an electronic state change from semiconducting to metallic observed upon alkali-metal ion's intercalation (4). Recent developments of in-situ transmission electron microscopy (TEM), as one unique tool to conduct real time structural measurements under the dynamic electrochemical reaction processes. (5) Such in-situ or in-operando measurements make it possible to analyze and tackle the intricacies of the sodiation mechanism in electrode materials during charge/discharge cycles.

In general, a large electron beam dose is required to imaging materials structure at atomic resolution, which introduces artificial microstructural changes during the observations. Herein, we applied in-situ electron diffraction with a remarkably low electron beam dosage to study the dynamic structural evolution in sodium intercalated $\mathrm{MoS}_{2}$ crystals during sodiation. The diffraction patterns are then carefully indexed and compared to the DFT calculations to derive the structural evolution at or close-to atomic resolution and unravel the mechanism of $\mathrm{MoS}_{2}$ sodiation under dynamic conditions.

Figure 1 shows the recorded electron diffraction patterns along with the sodiation process and the illustration of the Na-ion intercalated structures with $\mathrm{Na}$ content from 0.125 to 1.75 (the formula of each crystal is labeled). In each indexed pattern, the measured $d$-spacing of three spots are labeled. When $\mathrm{Na}$ content reaches 0.375 , phase transformation from $2 \mathrm{H}$ to $1 \mathrm{~T}$ occurs with appearance of superlattice spots. When $\mathrm{Na}$ content is higher than 1.75, the conversion reaction occurs, leading to the formation of metallic $\mathrm{Mo}$ and $\mathrm{Na}_{2} \mathrm{~S}$ reaction product. The structural models of the Na-intercalated structures are derived from DFT calculations and their simulated electron diffraction patterns are compared to the experimental ones; as shown in Fig.2. Several metastable phases have been identified in the early stage of sodiation in nanostructured $\mathrm{MoS}_{2}$ crystals. The nano-MoS 2 crystal can host up to $1.75 \mathrm{Na}$ per unit formula before the layered structure collapses. This study provides relevant insights into understanding the sodiation kinetics in typical layered transition-metal dichalcogenide structures, which we believe will be helpful in designing next generation advanced sodium ion batteries of the future [6].

References: 
[1] E. Benavente et al, Coordination Chemistry Reviews 224 (2002), p. 87.

[2] J. Xiao et al. Chemistry of Materials 22 (2010), p.4522.

[3] X. Wang et al, ACS Nano 8 (2014), p.11394.

[4] P. Gao et al, ACS Nano 9 (2015), p. 11296.

[5] J.Y. Huang et al, Science 330 (2010), p. 1515.

[6] This work was supported as part of the Center for Electrochemical Energy Science, an Energy Frontier Research Center funded by the U.S. Department of Energy (DOE), Office of Science, Basic Energy Sciences under Award \# DEAC02-06CH11357, and the Initiative for Sustainability and Energy at Northwestern (ISEN). This work was also supported by the NUANCE Center at Northwestern University, using the EPIC facility that receives support from the Soft and Hybrid Nanotechnology Experimental (SHyNE) Resource (NSF NNCI1542205); the MRSEC program (NSF DMR-1121262) at the Materials Research Center; the International Institute for Nanotechnology (IIN); the Keck Foundation; and the State of Illinois, through the IIN. We gratefully acknowledge the computing resources from: 1) the National Energy Research Scientific Computing Center, a DOE Office of Science User Facility supported by the Office of Science of the U.S. Department of Energy under Contract DE-AC02-05CH11231. 2) Blues, a high-performance computing cluster operated by the Laboratory Computing Resource Center at Argonne National Laboratory.

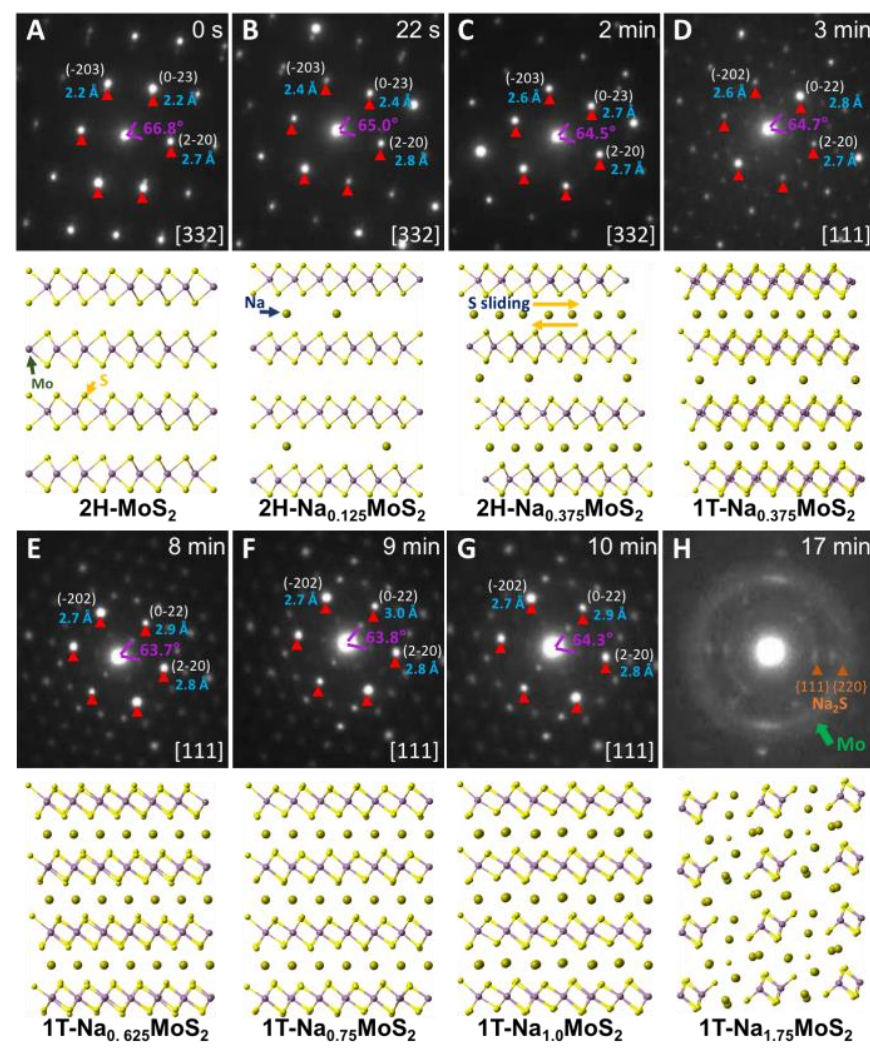

Figure 1. In-situ electron diffraction of Na-intercalation into $\mathrm{MoS}_{2}$. (A) The starting $2 \mathrm{H}-\mathrm{MoS}_{2}$ is identified to be oriented along the [332] zone axis, along with the illustration of the atomic structure (underneath the pattern). (B)-(H) The indexed diffraction patterns and structural illustrations of Na-ion intercalated structures with $\mathrm{Na}$ content from 0.125 to 1.75 (the formula of each crystal is labeled), along with the sodiation, while the sodiation time is marked in each pattern. In each indexed pattern, the measured $d$-spacing of three spots are labeled. When $\mathrm{Na}$ content reaches 0.375, phase transformation from $2 \mathrm{H}$ to $1 \mathrm{~T}$ occurs with appearance of superlattice spots. When Na content is higher than 1.75, the conversion reaction occurs leading to the formation of $\mathrm{Mo}$ and $\mathrm{Na}_{2} \mathrm{~S}$.

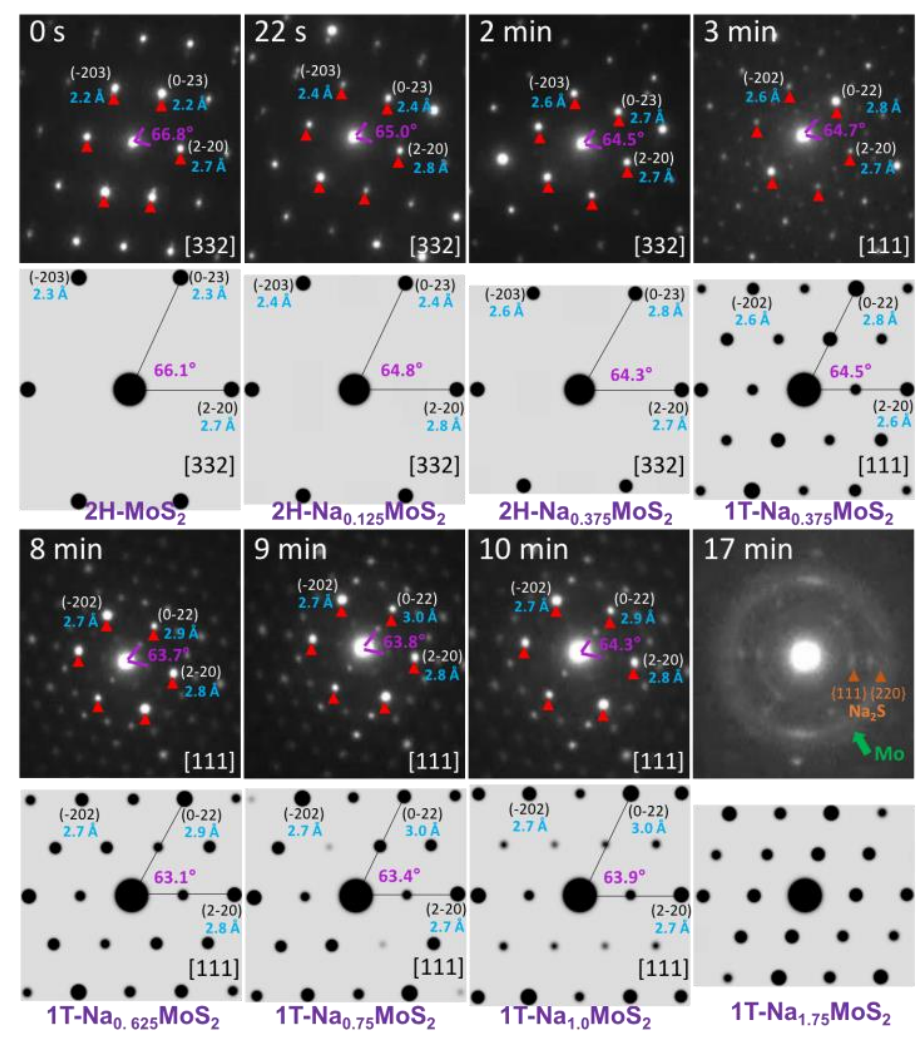

Figure 2. Comparison of experimental electron diffraction patterns to the simulated ones. The experimental selectedarea electron diffraction (SAED) patterns of all the intermediate phases appeared in the sodium insertion process are compared to the simulated ones calculated by DFT. 